\title{
GPER in CAFs regulates hypoxia-driven breast cancer invasion in a CTGF-dependent manner
}

\author{
JUAN REN $^{1}$, HUI GUO ${ }^{1}$, HUILI WU $^{1}$, TAO TIAN ${ }^{1}$, DANFENG DONG $^{1}$, YUELANG ZHANG $^{3}$, \\ YANXIA SUI ${ }^{4}$, YONG ZHANG ${ }^{5}$, DONGLI ZHAO ${ }^{1}$, SHUFENG WANG ${ }^{1}$, ZONGFANG LI $^{2}$, XIAOZHI ZHANG ${ }^{1}$, \\ RUI LIU ${ }^{1}$, JIANSHNEG QIAN ${ }^{1}$, HONGXIA WEI ${ }^{1}$, WENJUN JIANG ${ }^{6}$, YA LIU ${ }^{1}$ and YI LI ${ }^{1}$ \\ ${ }^{1}$ Cancer Center, First Affiliated Hospital of Xi'an Jiaotong University; ${ }^{2}$ Second Affiliated Hospital of Xi'an Jiaotong University; \\ Departments of ${ }^{3}$ Imaging, ${ }^{4}$ Pathology, First Affiliated Hospital of Xi'an Jiaotong University; ${ }^{5}$ Department of Orthopedics, \\ Xi'an Children Hospital; ${ }^{6}$ Medical School, Xi'an Jiaotong University, Xi'an, Shaanxi 710061, P.R. China
}

Received October 22, 2014; Accepted January 19, 2015

DOI: 10.3892/or.2015.3779

\begin{abstract}
Recent advances indicate that cancer-associated fibroblasts (CAFs) play a key role in cancer progression by contributing to invasion, metastasis and angiogenesis. Solid tumors often experience low oxygen tension environments, which induce gene expression changes and biological features leading to poor outcomes. The G-protein estrogen receptor (GPER) exhibits a stimulatory role in diverse types of cancer cells and in CAFs under hypoxic conditions. We investigated the role of CAFs and hypoxia in breast cancer aggressiveness, and examined the effect of GPER in CAFs on hypoxia-driven breast cancer progression. The results showed that hypoxia upregulated HIF-1 $\alpha$, GPER and $\alpha$-SMA expression in CAFs, and induced the secretion of Interleukin-6 (IL-6), vascular endothelial growth factor (VEGF) and connective tissue growth factor (CTGF) in CAFs. However, GPER silencing abrogated the above hypoxia-driven cytokine expression in CAFs. Moreover, knockdown of GPER in CAFs suppressed breast cancer cell invasion induced by CAF conditioned media (CM). Furthermore, GPER silencing in CAFs inhibited hypoxia-increased CTGF expression in CAFs and breast cancer cells cultured with CM from CAFs under hypoxic conditions. In addition, CTGF is responsible for the observed effects of GPER on CAFs activation and breast cancer invasion. Our findings further extend the molecular mechanisms through which the tumor microenvironment may contribute to cancer progression.
\end{abstract}

Correspondence to: Professor Yi Li, Cancer Center, First Affiliated Hospital of Medical College, Xi'an Jiaotong University, 277 West Yanta Road, Xi'an, Shaanxi 710061, P.R. China

E-mail: liyi_0712@163.com

Key words: carcinoma-associated fibroblasts, hypoxia, G-protein estrogen receptor, connective tissue growth factor, breast cancer

\section{Introduction}

Breast cancer has become the first common malignancy in women in developed and developing countries. Approximately 1.3 million women are diagnosed with breast cancer annually worldwide (1). Surgery is the important therapeutic method of breast cancer. Chemotherapy, radiotherapy and endocrine therapy are also important in breast cancer. Although comprehensive therapy has been previously employed, approximately 0.5 million women patients succumb to breast cancer annually due to recurrence, metastasis and resistance to therapy (2). Therefore, more effective therapeutic strategies are required to improve treatment outcomes for breast cancer patients.

Tumor cells do not exist in isolation during disease progression. The occurrence of an intense fibro-inflammatory reaction involving immune cells (3) and cancer-associated fibroblasts $(4,5)$ is a prominent pathologic feature of breast cancer (6). The cooperative interactions among tumor cells and reactive stroma strongly contribute to cancer development and progression $(7,8)$. Cancer-associated fibroblasts (CAFs) have been indicated as the main cell component of the tumor microenvironment involved in cancer initiation, invasion and metastasis $(9,10)$. In breast malignancies, CAFs exert a pivotal role in tumor progression and resistance to therapeutics through multiple mechanisms, including the stimulation of new blood vessels (11), mainly generated by a hypoxic tumor microenvironment (12-14). The mechanisms of cell sensing and adaptation to stressful environments are activated within the hypoxic tumor mass, leading to the growth and aggressiveness of malignant cells (15). Despite this finding, issues relating to maintenance of the tumor fibrotic microenvironment during disease development remain to be addressed.

Solid tumors often experience low oxygen tension environments, which is predominantly caused by abnormal vasculature formation of the rapidly growing tumor mass. Tumor hypoxia is associated with enhanced tumor invasiveness, angiogenesis, and distant metastasis (16-18). GPER and HIF-1 $\alpha$ are recruited to the HRE site located within the VEGF promoter region and cooperatively act as a functional complex for the transcription of VEGF. Recent studies have shown that hypoxia induced GPER expression in breast cancer fibroblasts, 
and the cross-talk between HIF-1 $\alpha$ and GPER regulates the expression of the migratory factor CTGF (19).

We investigated the role of GPER in CAFs and examined the effect of GPER silencing on hypoxia-driven breast cancer progression. We found that GPER knockdown in CAFs suppressed hypoxia-induced CAF activation and breast cancer cell invasion through the inhibition of CTGF expression.

\section{Materials and methods}

Materials. The antibodies used in this study included polyclonal rabbit anti-human HIF-1 $\alpha$ (Bioworld, St. Louis Park, MN, USA), polyclonal rabbit anti-human CTGF (Santa Cruz Biotechnology, Inc., Santa Cruz, CA, USA), polyclonal rabbit anti-human anti-GPER (Santa Cruz Biotechnology, Inc.), monoclonal mouse anti-human MMP-9 (Santa Cruz Biotechnology, Inc.), polyclonal rabbit anti-human uPA (Bioworld), monoclonal mouse anti-human $\alpha$-SMA (Sigma, St. Louis, MO, USA), monoclonal mouse anti-human cytokeratin14 (Sigma) and monoclonal mouse anti-human $\beta$-actin (Santa Cruz Biotechnology, Inc.).

Cell cultures. CAFs were extracted from invasive mammary ductal carcinomas obtained from mastectomies as previously described (20). These tissues were obtained from the Department of Cancer Center at the First Affiliated Hospital of Xi'an Jiaotong University. Signed informed consent from all the patients was obtained. The study protocol and consent forms were approved by the Ethics and Indications Committee of the First Affiliated Hospital of Medical College, Xi'an Jiaotong University, China. In particular, tissues obtained were cut into smaller sections (1-2 mm diameter), placed in digestion solution [400 IU collagenase, 100 IU hyaluronidase and 10\% FBS (HyClone, Logan, UT, USA), containing antibiotics and antimycotics solution] and incubated overnight at $37^{\circ} \mathrm{C}$. The cells were then separated by differential centrifugation at $90 \mathrm{x} \mathrm{g}$ for $2 \mathrm{~min}$.

The supernatant containing fibroblasts was centrifuged at $485 \mathrm{x}$ g for $8 \mathrm{~min}$, the pellet obtained was suspended in fibroblast growth medium (Medium 199 and Ham's F12 mixed 1:1 and supplemented with $10 \%$ FBS and $1 \%$ penicillin) and cultured at $37^{\circ} \mathrm{C}, 5 \% \mathrm{CO}_{2}$. CAFs were then expanded into two $15-\mathrm{cm}$ Petri dishes and stored as cells passaged for 2-3 population doublings within a total 7-10 days after tissue dissociation. CAFs were passaged for up to five population doublings for subsequent experiments to minimize clonal selection and culture stress, which could occur during extended tissue culture. Primary cell cultures of breast CAFs were characterized by immunofluorescence. Briefly, the cells were incubated with anti- $\alpha$-SMA and anti-cytokeratin14 (Fig. 1A), all antibodies were from Sigma. MDA-MB-231 human breast cancer cells were purchased from the Cell Bank of the Chinese Academy of Sciences (Shanghai, China) and were cultured at $37^{\circ} \mathrm{C}$ with $5 \% \mathrm{CO}_{2}$ and $95 \%$ air in L-15 (Sigma) containing $10 \%$ heat-inactivated fetal bovine serum (FBS) (HyClone) plus $100 \mu \mathrm{g} / \mathrm{ml}$ ampicillin and $100 \mu \mathrm{g} / \mathrm{ml}$ streptomycin.

Activation of CAFs. CAFs were cultured in regular growth medium to $80 \%$ confluence. The cells were incubated under normoxic or hypoxic $\left(3 \% \mathrm{O}_{2}\right)$ conditions in fresh serum- free medium for $12 \mathrm{~h}$ prior to the collection of conditioned media (CM).

Western blot analysis. CAFs or MDA-MB-231 cells $\left(1 \times 10^{6}\right)$ grown under our experimental conditions were lysed for $20 \mathrm{~min}$ on ice in $300 \mu l$ of RIPA lysis buffer [50 mM Tris- $\mathrm{HCl}(\mathrm{pH} 7.5)$, $150 \mathrm{mM} \mathrm{NaCl}, 1 \%$ Triton X-100, 2 mM EDTA, 1 mM sodium orthovanadate, $1 \mathrm{mM}$ phenylmethanesulfonyl-fluoride, $10 \mu \mathrm{g} /$ $\mathrm{ml}$ aprotinin, $10 \mu \mathrm{g} / \mathrm{ml}$ leupeptin]. Total proteins $(100 \mu \mathrm{g})$ were loaded onto SDS-PAGE gels, separated, and transferred onto PVDF membranes (Roche, Penzberg, Germany). The membranes were blocked with 5\% non-fat dry milk in TBST [10 mM Tris- $\mathrm{HCl}$ (pH 8.0), $150 \mathrm{mM} \mathrm{NaCl}, 0.05 \%$ Tween-20] and were subsequently incubated with primary antibodies overnight at $4^{\circ} \mathrm{C}$. After 5 washes of 10 min each in TBST, the membranes were incubated with HRP-conjugated secondary antibodies (1:5000, Santa Cruz Biotechnology, Inc.) for $2 \mathrm{~h}$ and subsequently washed again. The peroxidase reaction was performed using an enhanced chemiluminescence detection system to visualize the immunoreactive bands.

Cell invasion assay. A chamber-based invasion assay (Millipore, Billerica, MA, USA) was performed to evaluate breast cancer cell invasion. Briefly, the upper surface of the membrane was coated with matrigel (BD Biosciences, Franklin Lakes, NJ, USA). MDA-MB-231 cells $\left(1 \times 10^{5}\right)$ were resuspended in the upper chamber in serum-free medium and allowed to migrate towards a serum gradient $(10 \%)$ in the lower chamber for $24 \mathrm{~h}$. The medium was aspirated from the inside of the insert, and the non-invasive cells on the upper side were removed by scraping with a cotton swab. The membrane was fixed with $4 \%$ paraformaldehyde and stained with crystal violet. The number of migrating cells was counted on each membrane in 10 random fields and photographed at a magnification of x100. The values reported were the averages of triplicate experiments.

Reverse-transcription quantitative PCR assay (RT-qPCR). Total RNAs were extracted from CAFs or breast cancer cells using TRIzol reagent (Invitrogen, Carlsbad, CA, USA), and reverse transcription was performed using the PrimeScript RT Reagent kit (Takara, Dalian, China) according to the manufacturer's instructions. Real-time experiments were carried out using the iQ5 Multicolor Real-time PCR Detection System (Bio-Rad, Hercules, CA, USA) and a SYBR-Green PCR kit (Takara). The following PCR program was used: denaturation at $95^{\circ} \mathrm{C}$ for $30 \mathrm{sec}$, followed by 40 cycles consisting of denaturation at $95^{\circ} \mathrm{C}$ for $5 \mathrm{sec}$, annealing at $60^{\circ} \mathrm{C}$ for $30 \mathrm{sec}$, and extension at $72^{\circ} \mathrm{C}$ for $30 \mathrm{sec}$. A melting curve analysis was applied to assess the specificity of the amplified PCR products. The PCR primer sequences used were: HIF-1 $\alpha$ 5'-AAG TCTAGGGATGCAGCA-3' (forward) and 5'-CAAGATCA CCAGCATCATG-3' (reverse), GPER 5'-ACACACCTG GGTGGACACAA-3' (forward) and 5'-GGAGCCAGAAG CCACATCTG-3' (reverse), VEGF 5'-TGCAGATTATGCG GATCAAACC-3' (forward) and 5'-TGCATTCACATTTGT TGTGCTGTAG-3' (reverse), CTGF 5'-ACCTGTGGGATG GGCATCT-3' (forward) and 5'-CAGGCGGCTCTG CTTCTCTA-3' (reverse), IL-6 5'-AGTTCCTGCAGTCCAG CCTGAG-3' (forward) and 5'-TCAAACTGCATAGCCACTT TCC-3' (reverse), GAPDH 5'-ACCACAGTCCATGCCAT 


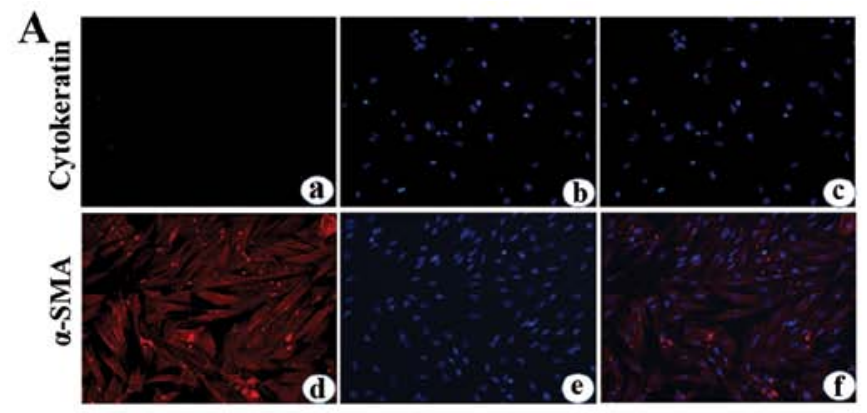

B

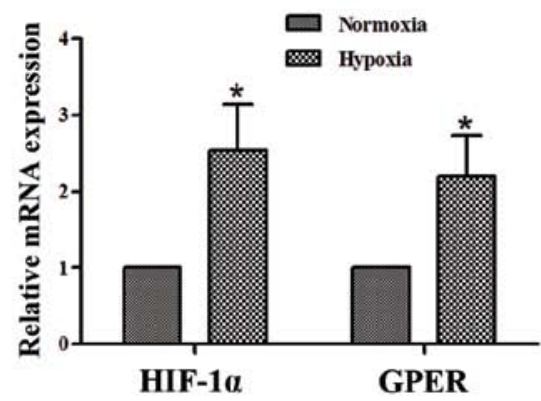

C

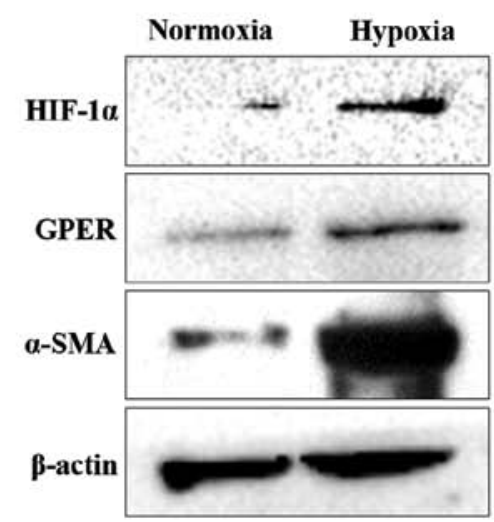

$\mathbf{D}$
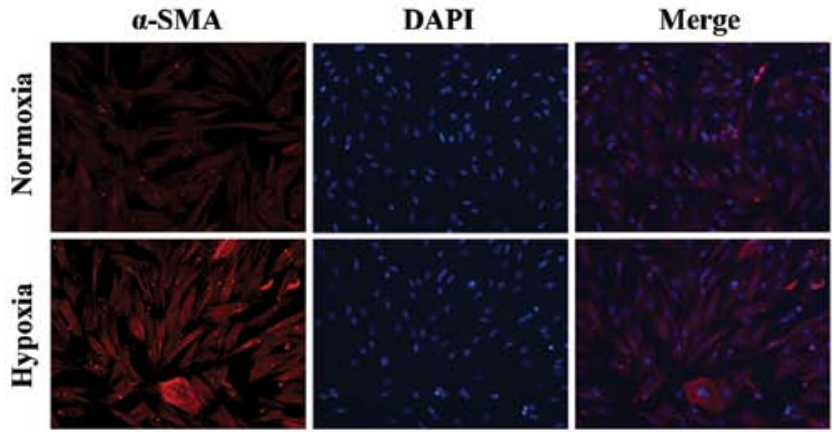

Figure 1. Hypoxia induces CAF activation and accumulates HIF-1 $\alpha$ and GPER expression. (A) CAFs were isolated from invasive mammary ductal carcinomas obtained from mastectomies. (a, b and c) Cytokeratin expression of CAFs was determined by immuofluorescence microscopy analysis. (d, e and f) $\alpha$-SMA expression of CAFs was determined by immuofluorescence microscopy analysis. (B) Subconfluent CAFs were treated with hypoxia for $12 \mathrm{~h}$ and serum-starved for an additional $24 \mathrm{~h}$. The cells were lysed, and HIF-1 $\alpha$ and GPER mRNAs were analyzed by RT-qPCR. ${ }^{*} \mathrm{P}<0.05$ vs. the normoxia group. (C) Subconfluent CAFs were treated with hypoxia for $12 \mathrm{~h}$ and serum-starved for an additional $24 \mathrm{~h}$. Immunoblots for HIF-1 $\alpha$, GPER, $\alpha$-SMA and $\beta$-actin were performed. (D) $\alpha$-SMA expression was analyzed in CAFs seeded on glass coverslips by immunofluorescence microscopy analysis. The data are representative of at least three independent experiments.

CAC-3' (forward) and 5'-TCCACCACCCTGTTGCTGAT-3' (reverse), The amount of each target gene was quantified by the comparative $\mathrm{C}(\mathrm{T})$ method using GAPDH as the normalization control (21).

Enzyme-linked immunosorbent assay (ELISA). The cells were conditioned in serum-free medium for $24 \mathrm{~h}$. The culture media were then collected and centrifuged at 1,500 rpm for $5 \mathrm{~min}$ to remove particles. The supernatants were then frozen at $-80^{\circ} \mathrm{C}$ until use. The production of CTGF, IL-6, and VEGF in the supernatants of CAFs was assessed by ELISA using a commercially available ELISA kit (R\&D Systems, Minneapolis, MN, USA) according to the manufacturer's instructions.

Immunofluorescence microscopy. After the designated treatment, CAFs were fixed with $4 \%$ paraformaldehyde for $10 \mathrm{~min}$ at room temperature, permeabilized in $0.5 \%$ Triton X-100 for $10 \mathrm{~min}$, and blocked in $1 \% \mathrm{BSA}$ for $1 \mathrm{~h}$. Fixed cells were then incubated with mouse anti-human- $\alpha$-SMA antibodies $(1: 100)$ or mouse anti-human-cytokeratin 14 antibodies $(1: 100)$ at $4{ }^{\circ} \mathrm{C}$ overnight. The cells were washed and incubated with goat antimouse dyelight 594 (red) IgG antibody (Qenshare Biological Inc., Xi'an, China) at 1:200 dilution for $60 \mathrm{~min}$. Nuclei were stained with DAPI for $5 \mathrm{~min}$. The cells were visualized by a fluorescent microscope (Observer A1, Carl Zeiss Microscopy $\mathrm{GmbH}$, Germany) using appropriate excitation and emission spectra at a magnification of $\mathrm{x} 400$.
RNA interference. siRNA against GPER (5'-CUGACACC GUCGACCAGGATT-3', 5'-UCCUGGUCGACGGUGUC GTT-3'), siRNA against CTGF (5'-AGAAUAUGAUGUUCA UCAATT-3', 5'-UUGAUGAACAUCAUAUUCUTT-3'), and a negative control siRNA (5'-UUCUCCGAACGUGUCAC GUTT-3', 5'-ACGUGACACGUUCGGAGAATT-3') were obtained from GenePharm (Shanghai, China). Cells $\left(2 \times 10^{5}\right.$ per well) were seeded in six-well plates and transfected with $100 \mathrm{nM}$ siRNA using Lipofectamine RNAiMAX reagent (Invitrogen) according to the manufacturer's instructions. After 48-h transfection, the cells were used for subsequent experiments.

Statistical analysis. The data are presented as the means \pm SD from at least three independent experiments. Statistical analysis of the data was performed using Student's t-test using SPSS software (version 13.0; SPSS, Chicago, IL, USA). P<0.05 was considered statistically significant.

\section{Results}

Hypoxia induces HIF-1 $\alpha$, GPER and $\alpha$-SMA expression in $C A F s$. Primary cell culture of breast CAFs was characterized by immunofluorescence. Briefly, the cells were incubated with human anti- $\alpha$-SMA and human anti-cytokeratin (Fig. 1A). To provide insight into the response to hypoxia in the main components of the tumor microenvironment such as CAFs, we showed that hypoxia induced the mRNA expression of HIF-1 $\alpha$ 


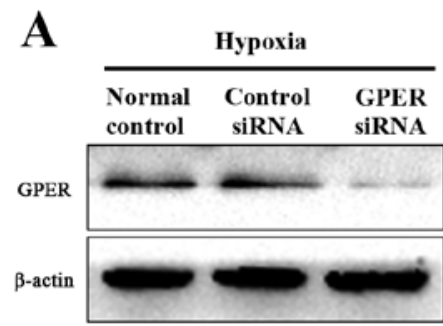

C

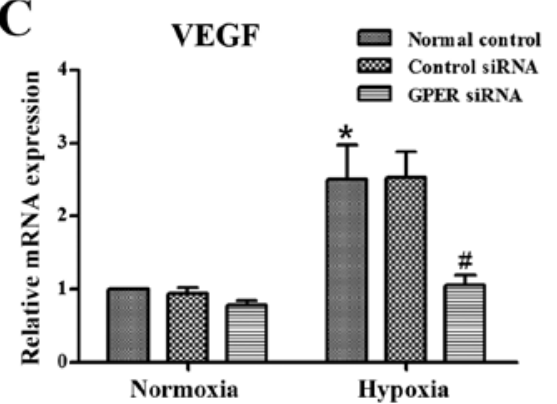

B

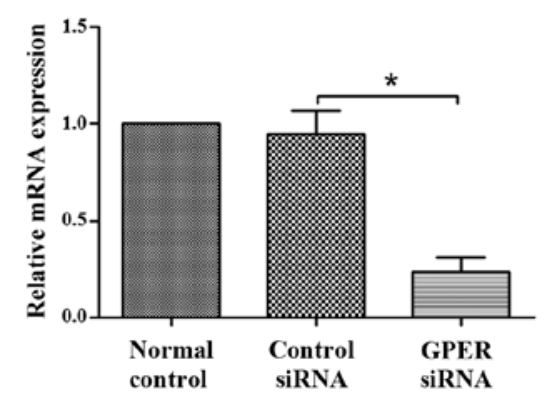

B IL-6

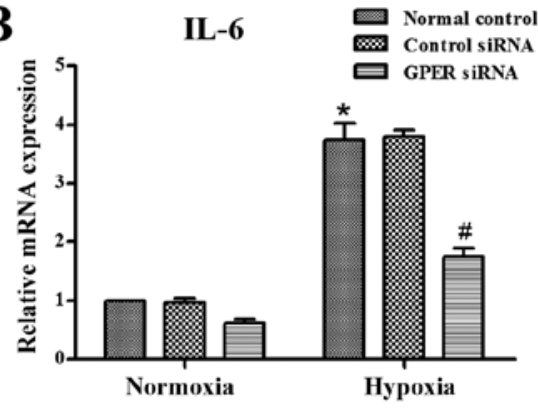

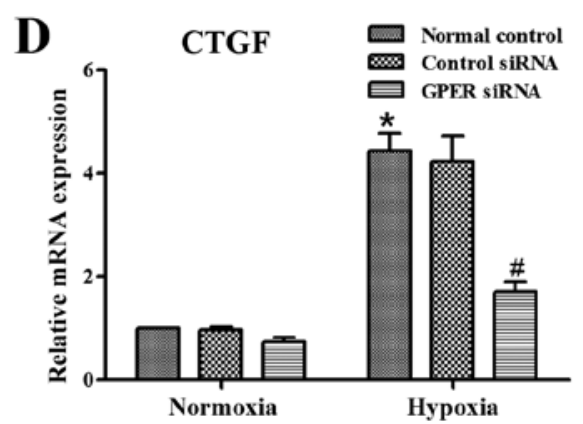

Figure 2. GPER knockdown eliminates the expression of IL-6, VEGF and CTGF in activated CAFs under hypoxia exposure. Following transfection with siRNA for $48 \mathrm{~h}$, CAFs were cultured under normoxic or hypoxic conditions for $12 \mathrm{~h}$. (A) GPER interference efficiency in CAFs under hypoxia was analyzed by western blotting (left) and RT-qPCR (right). ${ }^{*} \mathrm{P}<0.05$. CAFs were treated with hypoxia for $12 \mathrm{~h}$, and the cells were serum-starved for an additional $24 \mathrm{~h}$. (B) IL-6, (C) VEGF and (D) CTGF mRNAs were evaluated by RT-qPCR. ${ }^{*} \mathrm{P}<0.05$ vs. the normal control group under normoxia. ${ }^{\prime} \mathrm{P}<0.05$ vs. the control siRNA group under hypoxia. The data are representative of at least three independent experiments.

and its target gene GPER, as ascertained by qPCR (Fig. 1B). The induction of HIF- $1 \alpha$ and GPER mRNA expression was paralleled by increased protein levels of these factors in CAFs exposed to a low-oxygen tension $\left(3 \% \mathrm{O}_{2}\right)$ for $12 \mathrm{~h}$ (Fig. 1C). Furthermore, we observed that hypoxia increased activation of CAFs, as revealed by $\alpha$-SMA expression (Fig. 1C and D).

GPER silencing abrogates hypoxia-activated IL-6, VEGF and $C T G F$ secretion in $C A F S$. Previous results from other laboratories indicated that the activated stroma secretes large amounts of IL-6, VEGF and CTGF, leading to a significant increase in the invasive capacity of the surrounding tumor cells (22-24). To verify whether hypoxia-activated CAFs overexpress these soluble growth factors and cytokines, we performed RT-qPCR and ELISA to quantify IL-6, VEGF and CTGF expression. As shown in Fig. 2B-D and Fig. 3A-C, CAFs cultured under hypoxic conditions exhibit higher levels of IL-6, VEGF and CTGF transcription and secretion. These factors are known to be involved in modulating the response of tumor cells to activated CAFs. GPER was involved in hypoxia-induced VEGF expression in breast cancer CAFs (19). We investigated the role of GPER in these hypoxia-induced effects, and found that GPER was knocked down by siRNA (Fig. 2A). The data showed that silencing of GPER abrogated the hypoxia-induced overexpression of these factors in CAFs (Fig. 2B-D and Fig. 3A-C).

Knockdown of GPER in CAFs suppresses breast cancer cell invasion induced by CAF conditioned media under hypoxic conditions. It has been demonstrated that high levels of GPER expression in cancer cells are linked to enhanced invasive potential $(25,26)$. Thus, we investigated whether GPER derived from stromal components also influenced the behavior of tumor cells. We examined whether media from CAFs cultured under hypoxic conditions promoted the metastatic potential of cancer cells (using MDA-MB-231 cells derived from human primary breast adenocarcinoma). We treated MDA-MB-231 cells with conditioned media (CM) from CAFs activated by hypoxia with or without GPER silencing, and assayed their ability to express invasion-associated enzymes (e.g., MMP-9 or uPA) and to invade through a reconstituted matrigel barrier. The results revealed that $\mathrm{CM}$ from CAFs significantly increased the MMP-9 and uPA levels of breast cancer cells under either normoxic or hypoxic conditions (Fig. 4A). Moreover, CM from CAFs was mildly active in promoting the invasiveness of breast cancer cells under the two conditions (Fig. 4B and C). Exposure of CAFs to hypoxia during their activation enhances their ability to affect breast cancer motility, leading to a 1.7-fold (normoxia) or 2.2-fold (hypoxia) increase in invasiveness (Fig. 4B and C). However, GPER knockdown eliminated the effects of activated CAFs and hypoxia on breast cancer invasiveness (Fig. 4). These findings suggested that CAFs are sensitive to hypoxia, which enhances their promotion of breast cancer invasiveness.

GPER silencing in CAFs inhibits hypoxia-increased CTGF expression in CAFs and breast cancer cells cultured with $C M$ from CAFs. In tumor cells, CTGF has been reported to regulate growth, migration, invasion, and angiogenesis (27-29). We investigated whether CTGF is involved in the hypoxiadriven programs by GPER. We therefore analyzed CTGF expression in CAFs and breast cancer cells under normoxic and hypoxic conditions. In CAFs exposed to hypoxia, there 

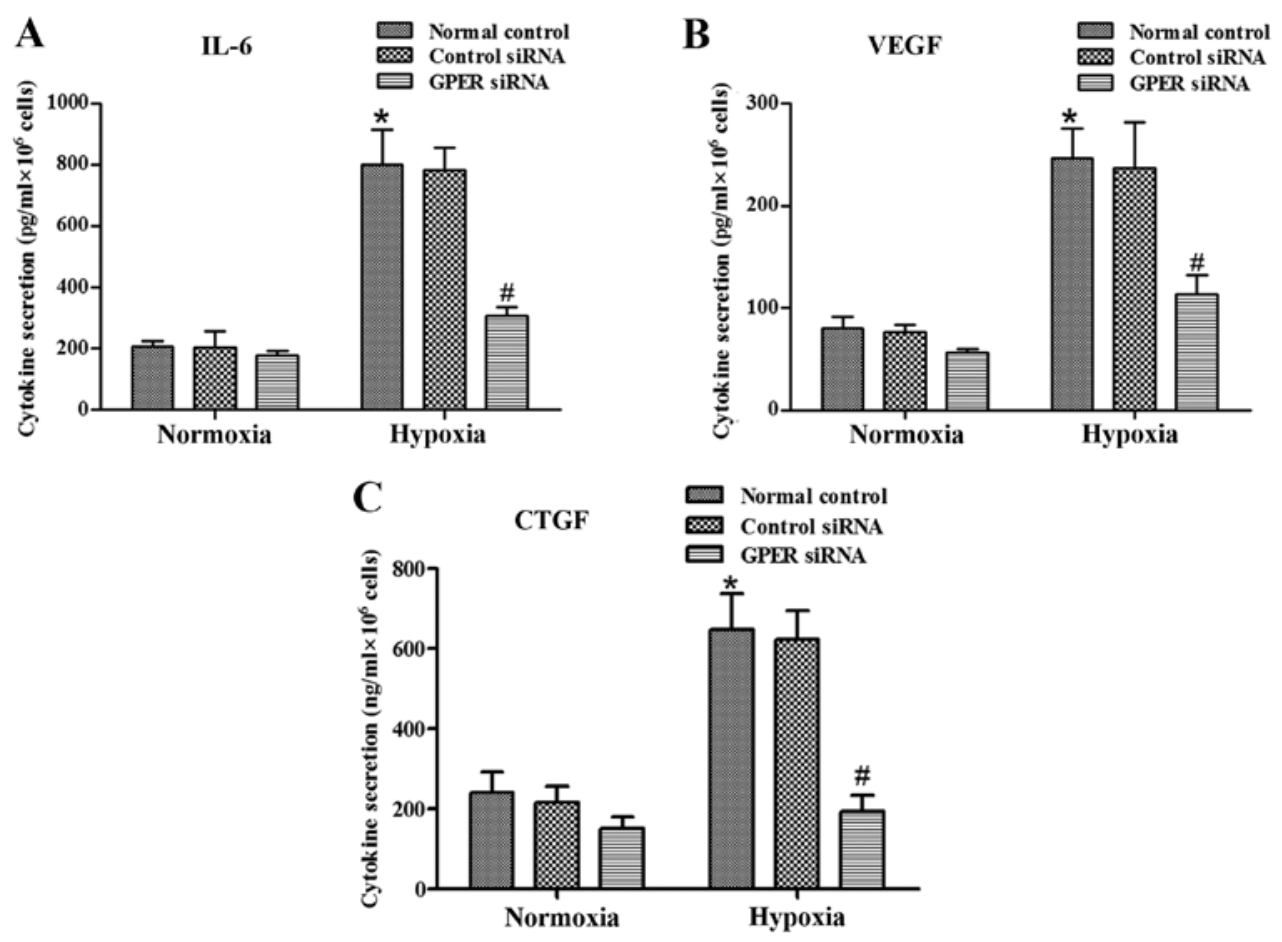

Figure 3. GPER knockdown abrogates IL-6, VEGF and CTGF in activated CAFs under hypoxia exposure. Following transfection with siRNA for $48 \mathrm{~h}$, CAFs were cultured under normoxic or hypoxic conditions for $12 \mathrm{~h}$. The cells were then serum-starved for an additional $24 \mathrm{~h}$. ELISA was performed to detect the expression of IL-6 (A), VEGF (B) and CTGF (C) in the CAF culture media as described in Materials and methods. "P $<0.05$ vs. the normal control group under normoxia. ${ }^{*} \mathrm{P}<0.05$ vs. the control siRNA group under hypoxia. The data are representative of at least three independent experiments.

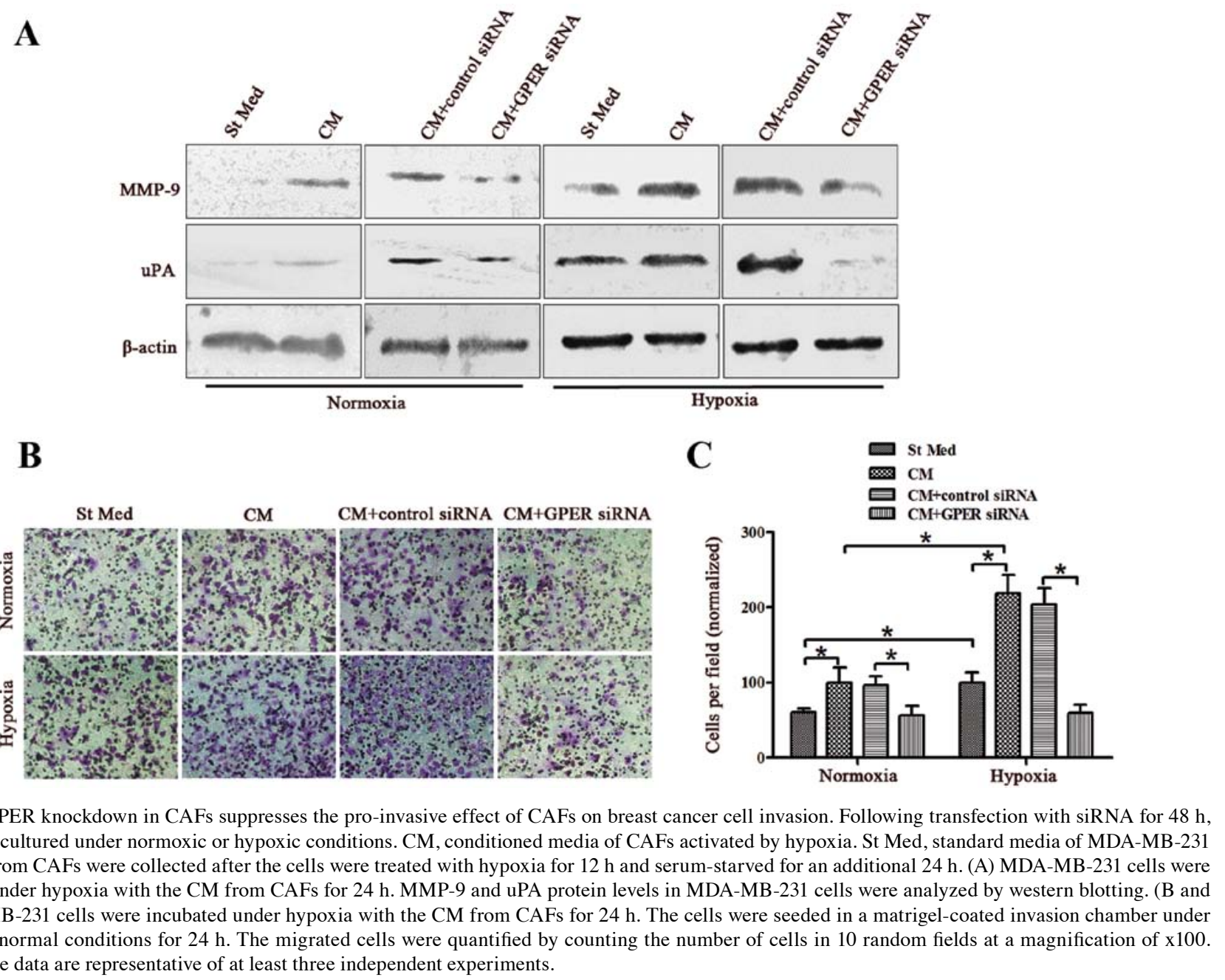

Figure 4. GPER knockdown in CAFs suppresses the pro-invasive effect of CAFs on breast cancer cell invasion. Following transfection with siRNA for $48 \mathrm{~h}$, CAFs were cultured under normoxic or hypoxic conditions. CM, conditioned media of CAFs activated by hypoxia. St Med, standard media of MDA-MB-231 cells. CM from CAFs were collected after the cells were treated with hypoxia for $12 \mathrm{~h}$ and serum-starved for an additional $24 \mathrm{~h}$. (A) MDA-MB-231 cells were incubated under hypoxia with the CM from CAFs for $24 \mathrm{~h}$. MMP-9 and uPA protein levels in MDA-MB-231 cells were analyzed by western blotting. (B and C) MDA-MB-231 cells were incubated under hypoxia with the CM from CAFs for $24 \mathrm{~h}$. The cells were seeded in a matrigel-coated invasion chamber under hypoxic or normal conditions for $24 \mathrm{~h}$. The migrated cells were quantified by counting the number of cells in 10 random fields at a magnification of $x 100$. ${ }^{*} \mathrm{P}<0.05$. The data are representative of at least three independent experiments. 


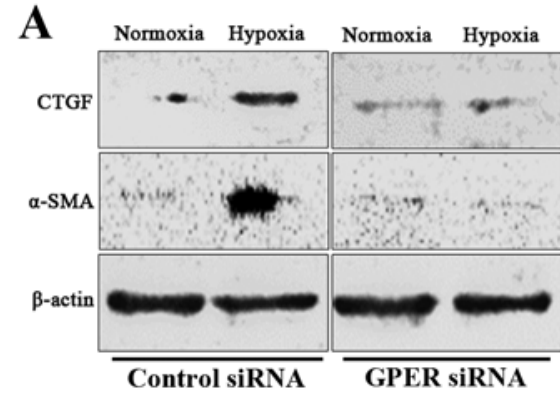

B

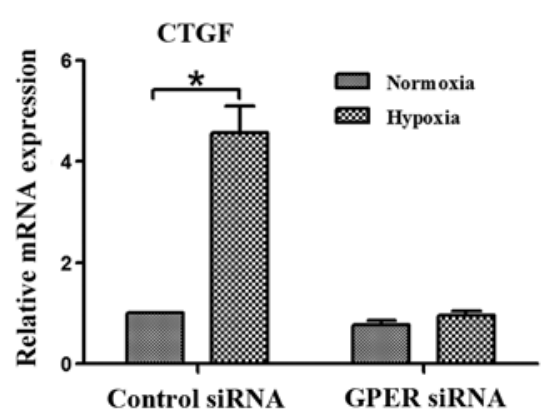

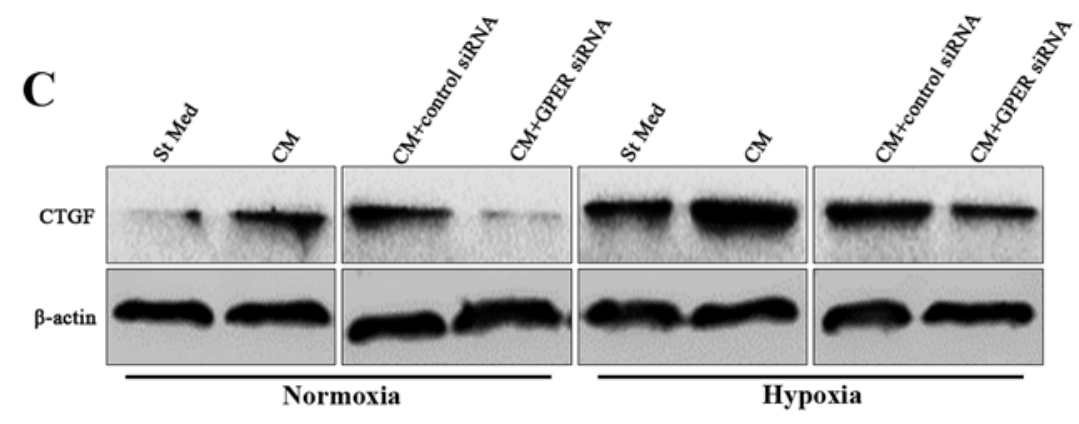

D

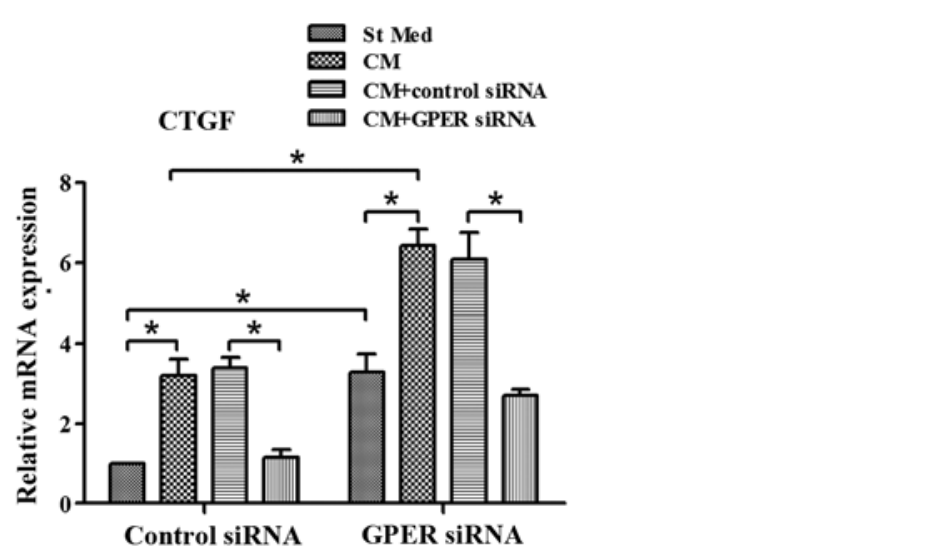

Figure 5. GPER silencing in CAFs attenuates hypoxia-induced CTGF upregulation in CAFs and breast cancer cells cultured with CM from CAFs. Following transfection with siRNA for 48 h, CAFs were cultured under normoxic or hypoxic conditions. CM, conditioned media of CAFs activated by hypoxia. St Med, standard media of MDA-MB-231 cells. (A and B) Subconfluent CAFs were treated with hypoxia for $12 \mathrm{~h}$ and serum-starved for an additional $24 \mathrm{~h}$. (A) CAFs were lysed, and immunoblots for CTGF and $\alpha$-SMA were performed. (B) mRNA expression of CTGF in CAFs was analyzed by RT-qPCR as described in Materials and methods. ${ }^{*}<0.05$. (C and D) MDA-MB-231 cells were incubated under hypoxia with or without the CM from CAFs for 24 h. (C) $C T G F$ protein levels of MDA-MB-231 cells were analyzed by western blotting. (D) mRNA expression of CTGF in MDA-MB-231 cells was analyzed by RT-qPCR. * $<0.05$. The data are representative of at least three independent experiments.

was a significant increase in CTGF expression, whereas CTGF exhibited a low expression under normoxic conditions (Fig. 5A and B). MDA-MB-231 cells were treated with CM from CAFs activated by hypoxia for $24 \mathrm{~h}$ under normoxic and hypoxic conditions. Hypoxia significantly increased CTGF expression in MDA-MB-231 cells, and treatment with CM from CAFs greatly increased this effect. Moreover, CM from CAFs was able to upregulate CTGF expression in MDA-MB-231 cells even under normoxic conditions (Fig. 5C and D). However, GPER silencing in CAFs abrogated CTGF expression in CAFs and breast cancer cells cultured with CM from CAFs under normoxic and hypoxic conditions (Fig. 5). Moreover, GPER knockdown eliminated CAF activation induced by hypoxia (Fig. 5A).

CTGF is responsible for the observed effects of GPER on $C A F$ activation and breast cancer invasion. Since GPER knockdown in CAFs may eliminate CTGF upregulation under hypoxia exposure in CAFs and MDA-MB-231 cells, we investigated whether CTGF is responsible for the observed effects of GPER on activation of CAFs and breast cancer invasion. CTGF siRNA was applied to the knockdown of CTGF expression in CAFs and MDA-MB-231 cells. Since the expression level of CTGF in CAFs under normoxic conditions was extremely low, we detected the interference efficiency of CTGF siRNA in CAFs and MDA-MB-231 cells under hypoxic conditions (Fig. 6A). The GPER and $\alpha$-SMA expression in CAFs and the MMP-9 and UPA expression in MDA-MB-231 cells were then examined. CTGF siRNA significantly suppressed $\alpha$-SMA expression in CAFs under normoxic and hypoxic conditions (Fig. 6B). However, GPER expression was not affected by CTGF siRNA (Fig. 6B and C). Moreover, knockdown of CTGF in MDA-MB-231 cells decreased the MMP-9 and UPA expression of MDA-MB-231 cells cultured with CM from hypoxia-activated CAFs under the two conditions (Fig. 6D). Since CTGF siRNA did not influence GPER expression in CAFs, and GPER knockdown downregulated CTGF expression, these data indicated that $C T G F$ is a downstream gene of GPER, and is responsible for the observed effects of GPER on CAFs activation and breast cancer invasion.

\section{Discussion}

The results of this study are consistent with a mandatory role for some components of the tumor microenvironment, i.e., CAFs and hypoxia, in the progression of breast cancer towards an aggressive phenotype. We provide evidence that i) stromal reactivity depends on hypoxia (particularly on its associated GPER expression); ii) hypoxia and activated CAFs exhibit synergy in promoting breast cancer invasiveness, increasing CTGF expression.

Breast tumors are characterized by an extensive desmoplastic stroma, abundantly populated by fibroblasts, and CAFs were shown to support the growth of mammary tumors (30). Accumulating evidence indicates that tumor desmoplasia plays a central role in disease progression and that activated CAFs 
$\mathbf{A}$
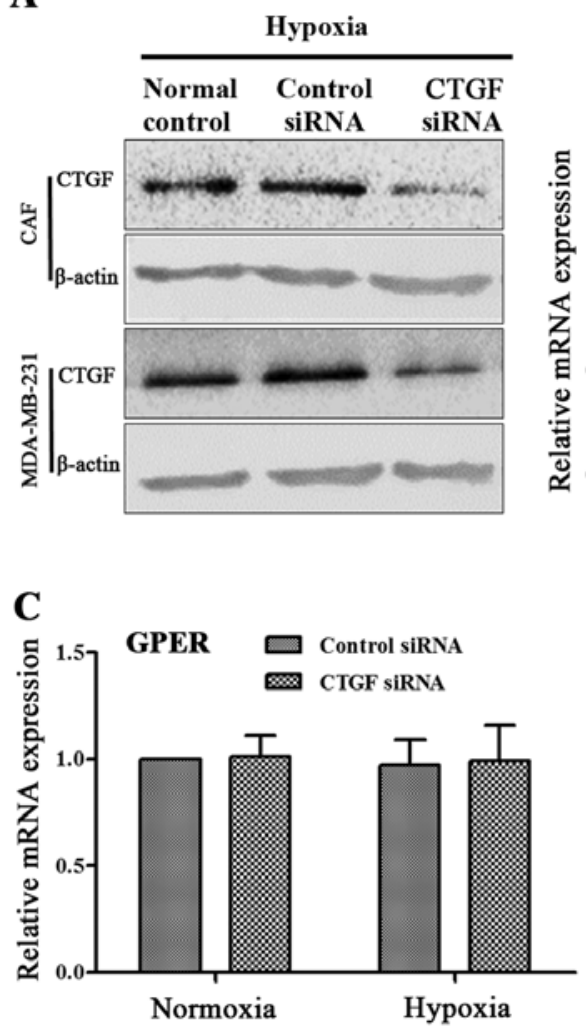
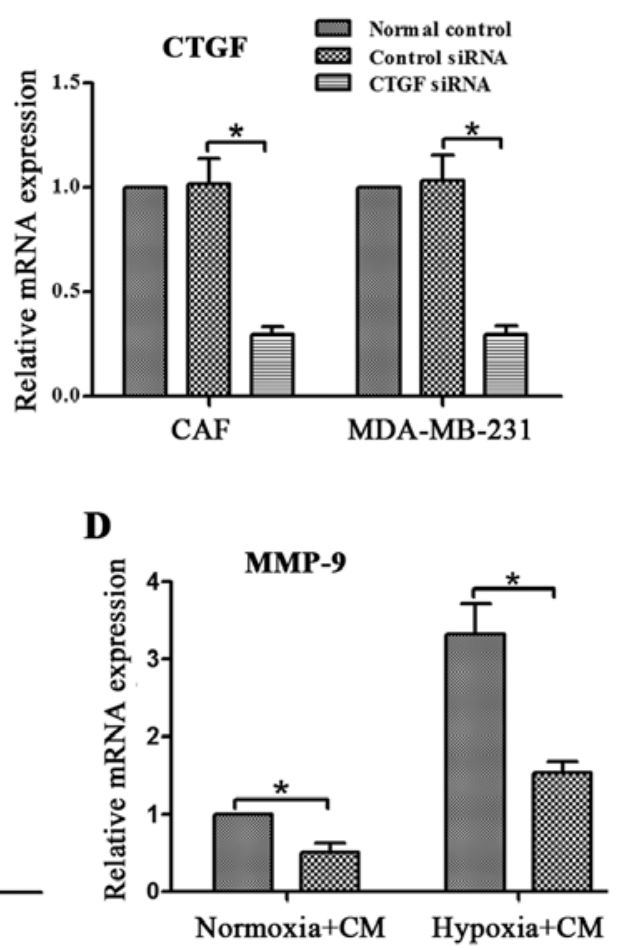

B
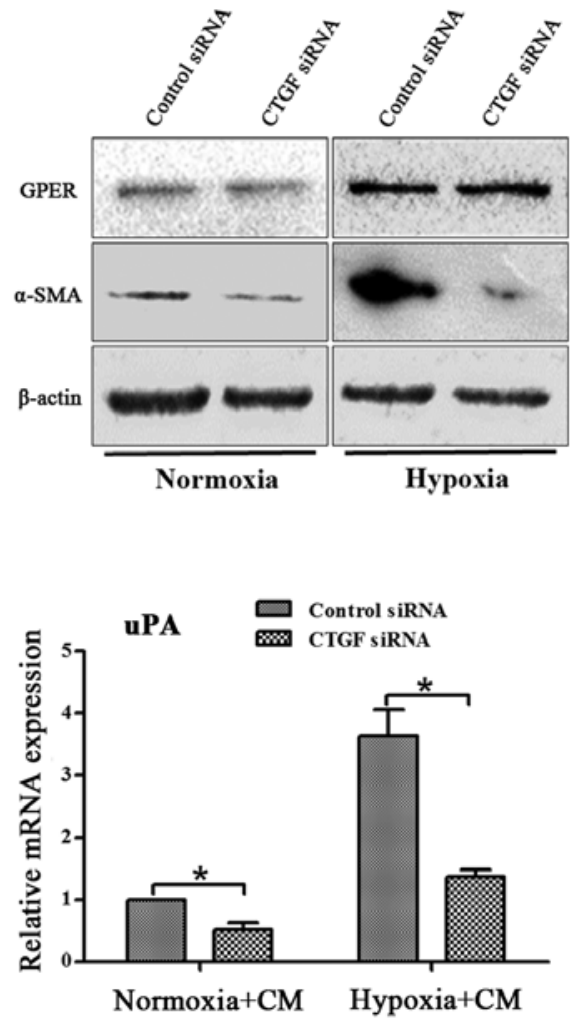

Figure 6. CTGF interference abrogates the observed effects of GPER silencing on CAFs activation and breast cancer invasion. (A) CTGF interference efficiency in CAFs and MDA-MB-231 cells under hypoxia were analyzed by western blotting (left) and RT-qPCR (right). (B and C) CAFs transfected with siRNA were cultured under normoxic or hypoxic conditions for $12 \mathrm{~h}$ and serum-starved for an additional $24 \mathrm{~h}$. (B) The GPER and $\alpha$-SMA protein level of CAFs were analyzed by western blotting. (C) The GPER mRNA level of CAFs was analyzed by RT-qPCR. (D) MDA-MB-231 cells transfected with siRNA were incubated with the conditioned media (CM) from CAFs under normoxia or hypoxia for $24 \mathrm{~h}$. The cells were lysed, and MMP-9 and uPA mRNAs were analyzed by RT-qPCR. "P $<0.05$. The data are representative of at least three independent experiments.

are responsible for the excess matrix production. The mechanisms underlying the interplay between tumor and stroma are complex. Various growth factors, such as transforming growth factor (TGF)- $\alpha$, TGF- $\beta$, insulin-like growth factor (IGF)-I, IGF-II and platelet-derived growth factor (PDGF), have been identified. These growth factors secreted by cancer cells and can stimulate stromal cells (31-33), which mediate effects on tumor growth, invasion, metastasis, and resistance to chemotherapy. It is therefore conceivable that the different stromal frameworks they encounter in this pathway grossly affect their behavior and their terminal differentiation. This result is consistent with our observation that MDA-MB-231 cells sense activated stromal CAFs with a clear increase in their invasiveness. This behavior of breast cancer cells in response to their activated CAF counterparts is common among other tumors, such as melanoma, pancreatic carcinoma, and prostate carcinoma, the motility and aggressiveness of which is enhanced following contact with CAFs (24,34-36). In addition, activated stromal prostate fibroblasts induce stem-like characteristics in carcinoma cells, thereby strengthening the effect of these fibroblasts on metastatic tumor growth $(35,37,38)$.

Solid tumors often experience low-oxygen tension, which is predominantly caused by abnormal vasculature formation in the rapidly growing tumor mass. Our data indicate that hypoxia activated CAFs and elicited the secretion of key cytokines such as VEGF, IL-6, and CTGF, which are known to exert angiogenic and inflammatory functions. Tumor hypoxia is recognized as a key factor in tumor progression in several cancer models, as it is correlated with de novo angiogenesis and with profound changes in tumor metabolism as well as achievement of motile behavior $(39,40)$. These events synergistically facilitate the metastatic spread of aggressive cells. Recent studies on breast cancer have shown that GPER is an HIF-1-regulated gene, which contributes to adaptation to a low-oxygen environment in breast cancer cells and in cardiomyocytes (41).

Our results suggest that CAFs also sense hypoxia through GPER upregulation, as GPER siRNA knockdown efficiently abolishes CAF activation and the effects of CAFs on breast cancer cells. As expected, GPER knockdown abolished the expression of VEGF, IL-6, and CTGF induced by activated CAFs, suggesting a key role for hypoxia-driven GPER expression in the regulation of angiogenic and inflammatory responses during breast cancer progression. Recchia et al recently reported that hypoxia leads to the upregulation of CTGF (41), which is a target gene of HIF-1 $\alpha(41,42)$ and GPER $(19,43)$. We also showed that CTGF participates in hypoxia-driven GPERinduced effects on CAFs and breast cancer cells.

VEGF and CTGF, which are involved in angiogenesis and invasion of cancer and endothelial cells, and IL-6, which is involved in the organization of the pro-inflammatory response, have already been reported to be under the transcriptional control of HIF-1 $(44,45)$. We have shown that in breast cancer, the secretion of these cytokines by activated CAFs is 
dependent on concomitant exposure to hypoxia. These results indicate that activated CAFs exposed to hypoxia are active players in attracting breast cancer cells to different locations. Active factors in this chemoattraction include CTGF, VEGF, and IL-6, confirming their pleiotropic role in breast cancer progression. Thus, the surrounding stroma, with intralesional hypoxic areas, may play a role in attracting metastatic breast cancer cells from the primary lesions, thereby facilitating satellite metastases.

GPER and HIF-1 $\alpha$ are recruited to the HRE site located within the VEGF promoter region and cooperatively act as a functional complex for the transcription of VEGF (19). The present results show that GPER knockdown abrogated hypoxiadriven CAF activation. Moreover, GPER silencing inhibited breast cancer cell invasion induced by CAF CM, and abolished hypoxia-activated CTGF, VEGF, and IL-6 secretion in CAFs. Additionally, GPER knockdown suppressed hypoxia-enhanced CTGF expression in CAFs and breast cancer cells cultured with CM from CAFs. However, siRNA-mediated downregulation of CTGF abolished the effects of GPER silencing on inhibiting CAF activation and breast cancer invasion. These data indicate that GPER silencing has a protective effect against hypoxia in the breast tumor-stromal interaction, which is associated with its ability to ameliorate CTGF upregulation.

\section{Acknowledgements}

This study is supported by the National Natural Science Foundations of China (NSFC) (Nos. 31201060/C0709, 30973175/H1621, 81172490/H1621), Program for New Century Excellent Talents in University (NCET-12-0440), Scientific and Technological Research Foundation of Shaanxi Province (Nos. 2012K13-01-06, 2007K09-09), Project sponsored by Scientific Research Foundation for the Returned overseas Chinese Scholars of State Education Ministry (0601-18920006), Research Foundation of Health Department of Shaan'xi Province (No. 2010D41), Qing Nian Jiao Shi Gen Zong Ji Hua of Xi'an Jiaotong University ('The Fundamental Research Funds for the Central Universities') (J.R., 2012), Program for Changjiang Scholars and Innovative Research Team in University (PCSIRT:1171), and the Research Fundation of Xi'an Jiao Tong University of China (J.R.).

\section{References}

1. Siegel R, Ma J, Zou Z and Jemal A: Cancer statistics, 2014. CA Cancer J Clin 64: 9-29, 2014.

2. Youlden DR, Cramb SM, Dunn NA, Muller JM, Pyke CM and Baade PD: The descriptive epidemiology of female breast cancer: an international comparison of screening, incidence, survival and mortality. Cancer Epidemiol 36: 237-248, 2012.

3. Clark CE, Hingorani SR, Mick R, Combs C, Tuveson DA and Vonderheide RH: Dynamics of the immune reaction to pancreatic cancer from inception to invasion. Cancer Res 67: 9518-9527, 2007.

4. Yen TW, Aardal NP, Bronner MP, Thorning DR, Savard CE, Lee SP and Bell RH Jr: Myofibroblasts are responsible for the desmoplastic reaction surrounding human pancreatic carcinomas. Surgery 131: 129-134, 2002.

5. Hwang RF, Moore T, Arumugam T, Ramachandran V, Amos KD, Rivera A, Ji B, Evans DB and Logsdon CD: Cancer-associated stromal fibroblasts promote pancreatic tumor progression. Cancer Res 68: 918-926, 2008

6. Neesse A, Michl P, Frese KK, et al: Stromal biology and therapy in pancreatic cancer. Gut 60: 861-868, 2011.
7. Polyak K and Kalluri R: The role of the microenvironment in mammary gland development and cancer. Cold Spring Harb Perspect Biol 2: a003244, 2010.

8. Bhowmick NA, Neilson EG and Moses HL: Stromal fibroblasts in cancer initiation and progression. Nature 432: 332-337, 2004.

9. Kalluri R and Zeisberg M: Fibroblasts in cancer. Nat Rev Cancer 6: 392-401, 2006.

10. Gaggioli C, Hooper S, Hidalgo-Carcedo C, Grosse R, Marshall JF, Harrington K and Sahai E: Fibroblast-led collective invasion of carcinoma cells with differing roles for RhoGTPases in leading and following cells. Nat Cell Biol 9: 1392-1400, 2007.

11. Shimoda M, Mellody KT and Orimo A: Carcinoma-associated fibroblasts are a rate-limiting determinant for tumour progression. Semin Cell Dev Biol 21: 19-25, 2010.

12. Liao D and Johnson RS: Hypoxia: a key regulator of angiogenesis in cancer. Cancer Metastasis Rev 26: 281-290, 2007.

13. Harris AL: Hypoxia - a key regulatory factor in tumour growth. Nat Rev Cancer 2: 38-47, 2002.

14. Rapisarda A and Melillo G: Role of the hypoxic tumor microenvironment in the resistance to anti-angiogenic therapies. Drug Resist Updat 12: 74-80, 2009.

15. Lundgren K, Holm C and Landberg G: Hypoxia and breast cancer: prognostic and therapeutic implications. Cell Mol Life Sci 64: 3233-3247, 2007.

16. Michieli P: Hypoxia, angiogenesis and cancer therapy: to breathe or not to breathe. Cell Cycle 8: 3291-3296, 2009.

17. Lei J, Ma J, Ma Q, et al: Hedgehog signaling regulates hypoxia induced epithelial to mesenchymal transition and invasion in pancreatic cancer cells via a ligand-independent manner. Mol Cancer 12: 66, 2013.

18. Lei J, Huo X, Duan W, et al: $\alpha$-Mangostin inhibits hypoxiadriven ROS-induced PSC activation and pancreatic cancer cell invasion. Cancer Lett 347: 129-138, 2014.

19. De Francesco EM, Lappano R, Santolla MF, Marsico S, Caruso A and Maggiolini M: HIF-1alpha/GPER signaling mediates the expression of VEGF induced by hypoxia in breast cancer associated fibroblasts (CAFs). Breast Cancer Res 15: R64, 2013.

20. Madeo A and Maggiolini M: Nuclear alternate estrogen receptor GPR30 mediates 17beta-estradiol-induced gene expression and migration in breast cancer-associated fibroblasts. Cancer Res 70: 6036-6046, 2010.

21. Schmittgen TD and Livak KJ: Analyzing real-time PCR data by the comparative C(T) method. Nat Protoc 3: 1101-1108, 2008.

22. Arias-Pulido H, Chaher N, Gong Y, Qualls C, Vargas J and Royce M: Tumor stromal vascular endothelial growth factor A is predictive of poor outcome in inflammatory breast cancer. BMC Cancer 12: 298, 2012.

23. Staton CA, Reed MW and Brown NJ: A critical analysis of current in vitro and in vivo angiogenesis assays. Int J Exp Pathol 90: 195-221, 2009.

24. Comito G, Giannoni E, Di GP, Segura CP, Gerlini G and Chiarugi P: Stromal fibroblasts synergize with hypoxic oxidative stress to enhance melanoma aggressiveness. Cancer Lett 324: 31-41, 2012.

25. Yu T, Liu M, Luo H, Wu C, Tang X, Tang S, Hu P, Yan Y, Wang Z and Tu G: GPER mediates enhanced cell viability and motility via non-genomic signaling induced by $17 \beta$-estradiol in triplenegative breast cancer cells. J Steroid Biochem Mol Biol 143: 392-403, 2014.

26. Jiang QF, Wu TT, Yang JY, Dong CR, Wang N, Liu XH and Liu ZM: $17 \beta$-estradiol promotes the invasion and migration of nuclear estrogen receptor-negative breast cancer cells through cross-talk between GPER1 and CXCR1. J Steroid Biochem Mol Biol 138: 314-324, 2013.

27. Dhar A and Ray A: The CCN family proteins in carcinogenesis. Exp Oncol 32: 2-9, 2010.

28. Chu CY, Chang CC, Prakash E and Kuo ML: Connective tissue growth factor (CTGF) and cancer progression. J Biomed Sci 15: 675-685, 2008 .

29. Hall-Glenn F, De Young RA, Huang BL, et al: CCN2/connective tissue growth factor is essential for pericyte adhesion and endothelial basement membrane formation during angiogenesis. PLoS One 7: e30562, 2012.

30. Orimo A, GuptaPB,Sgroi DC, Arenzana-Seisdedos F, Delaunay T, Naeem R, Carey VJ, Richardson AL and Weinberg RA: Stromal fibroblasts present in invasive human breast carcinomas promote tumor growth and angiogenesis through elevated SDF-1/CXCL12 secretion. Cell 121: 335-348, 2005. 
31. Ronnov-Jessen L and Petersen OW: Induction of alpha-smooth muscle actin by transforming growth factor-beta 1 in quiescent human breast gland fibroblasts. Implications for myofibroblast generation in breast neoplasia. Lab Invest 68: 696-707, 1993.

32. Ellis MJ, Singer C, Hornby A, Rasmussen A and Cullen KJ: Insulin-like growth factor mediated stromal-epithelial interactions in human breast cancer. Breast Cancer Res Treat 31: 249-261, 1994

33. Bronzert DA, Pantazis P, Antoniades HN, Kasid A, Davidson N Dickson RB and Lippman ME: Synthesis and secretion of platelet-derived growth factor by human breast cancer cell lines. Proc Natl Acad Sci USA 84: 5763-5767, 1987.

34. Taddei ML, Giannoni E, Raugei G, Scacco S, Sardanelli AM, Papa S and Chiarugi P: Mitochondrial oxidative stress due to complex dysfunction promotes fibroblast activation and melanoma cell invasiveness. J Signal Transduct 2012: 684592, 2012.

35. Giannoni E, Bianchini F, Masieri L, Serni S, Torre E, Calorini L and Chiarugi P: Reciprocal activation of prostate cancer cells and cancer-associated fibroblasts stimulates epithelial-mesenchymal transition and cancer stemness. Cancer Res 70: 6945-6956, 2010

36. Cirri P and Chiarugi P: Cancer-associated-fibroblasts and tumour cells: a diabolic liaison driving cancer progression. Cancer Metastasis Rev 31: 195-208, 2012.

37. Giannoni E, Bianchini F, Calorini L and Chiarugi P: Cancer associated fibroblasts exploit reactive oxygen species through a proinflammatory signature leading to epithelial mesenchymal transition and stemness. Antioxid Redox Signal 14: 2361-2371, 2011.
38. Mani SA, Guo W, Liao MJ, et al: The epithelial-mesenchymal transition generates cells with properties of stem cells. Cell 133: 704-715, 2008

39. Giaccia AJ and Schipani E: Role of carcinoma-associated fibroblasts and hypoxia in tumor progression. Curr Top Microbiol Immunol 345: 31-45, 2010.

40. Melillo G: Targeting hypoxia cell signaling for cancer therapy. Cancer Metastasis Rev 26: 341-352, 2007.

41. Recchia AG, De Francesco EM, Vivacqua A, Sisci D, Panno ML, Ando $\mathrm{S}$ and Maggiolini M: The G protein-coupled receptor 30 is up-regulated by hypoxia-inducible factor-1alpha (HIF-1alpha) in breast cancer cells and cardiomyocytes. J Biol Chem 286: 10773-10782, 2011.

42. Lappano R, Recchia AG, De Francesco EM, Angelone T, Cerra MC, Picard D and Maggiolini M: The cholesterol metabolite 25-hydroxycholesterol activates estrogen receptor alpha-mediated signaling in cancer cells and in cardiomyocytes. PLoS One 6: e16631, 2011.

43. Pandey DP, Lappano R, Albanito L, Madeo A, Maggiolini M and Picard D: Estrogenic GPR30 signalling induces proliferation and migration of breast cancer cells through CTGF. EMBO J 28: 523-532, 2009.

44. Semenza GL: Oxygen homeostasis. Wiley Interdiscip Rev Syst Biol Med 2: 336-361, 2010.

45. Youn SW, Lee SW, Lee J, et al: COMP-Ang1 stimulates HIF-1 $\alpha$ mediated SDF-1 overexpression and recovers ischemic injury through BM-derived progenitor cell recruitment. Blood 117: 4376-4386, 2011 